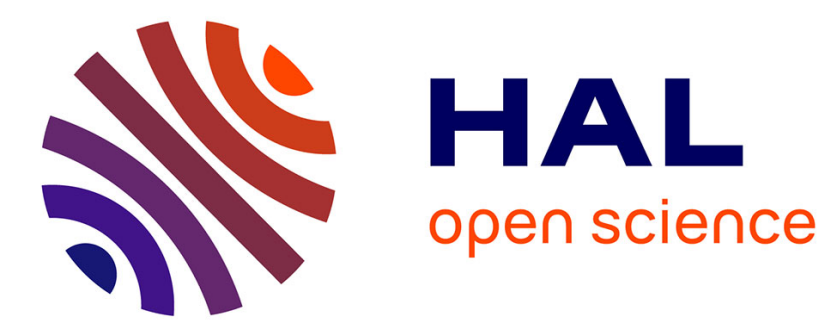

\title{
Some remarks on the connection between replicas and dynamics
}

\author{
G. Parisi
}

\section{To cite this version:}

G. Parisi. Some remarks on the connection between replicas and dynamics. Journal de Physique Lettres, 1983, 44 (14), pp.581-583. 10.1051/jphyslet:019830044014058100 . jpa-00232234

\section{HAL Id: jpa-00232234 https://hal.science/jpa-00232234}

Submitted on 1 Jan 1983

HAL is a multi-disciplinary open access archive for the deposit and dissemination of scientific research documents, whether they are published or not. The documents may come from teaching and research institutions in France or abroad, or from public or private research centers.
L'archive ouverte pluridisciplinaire HAL, est destinée au dépôt et à la diffusion de documents scientifiques de niveau recherche, publiés ou non, émanant des établissements d'enseignement et de recherche français ou étrangers, des laboratoires publics ou privés. 


\title{
Some remarks on the connection between replicas and dynamics
}

\author{
G. Parisi \\ Università di Roma II, Tor Vergata, Italy \\ and \\ Laboratori Nazionali INFN, Frascati, Italy
}

(Reçu le 9 mars 1983, accepté le 26 mai 1983)

Résumé. - Nous obtenons des prédictions sur l'évolution dans le temps d'un système de verres de spin en utilisant l'interprétation du paramètre d'ordre tout récemment proposée.

Abstract. - We use the recently proposed interpretation of the order parameter for spin glasses of the replica approach to obtain some predictions on the time evolution.

In the mean-field theory for spin glasses the order parameter is a function defined on the interval $0-1$ : in the glassy phase, where the linear response theory breaks down, the function $q(x)$ is not a constant [1].

The interpretation of the function $q(x)$ has been obtained quite recently; if we define the overlap between two configurations as [2]

$$
q^{(1,2)}=\frac{1}{N} \sum_{i=1}^{N} \sigma_{i}^{(1)} \sigma_{i}^{(2)}
$$

the probability distribution of $q(P(q))$ in the infinite volume limit is given by $\left({ }^{1}\right)$

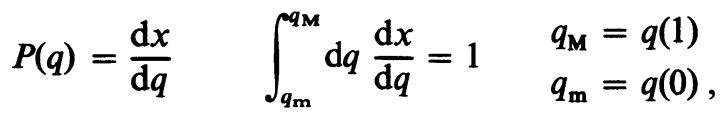

where $x(q)$ is the inverse function of $q(x)$.

Indeed it has been shown that if we consider two real replicas $\sigma_{i}$ and $\mu_{i}$ of the same system $\left(H\left(\sigma_{i}\right)=H\left(\mu_{i}\right)\right)$ we have that :

$$
\left\langle\exp \left(y \frac{1}{N} \sum_{i} \sigma_{i} \mu_{i}\right)\right\rangle=\int_{0}^{1} \mathrm{~d} x \exp (y q(x))=\int_{q_{\mathrm{m}}}^{q_{\mathrm{M}}} \mathrm{d} q \frac{\mathrm{d} x}{\mathrm{~d} q} \exp (y q) .
$$

( ${ }^{1}$ ) A similar equivalent, but slightly more complex formula holds in the approach of reference 3, where two order parameters $(q(x)$ and $\Delta(x))$ are present. 
Developing (3) in powers of $y$ and identifying the corresponding terms we easily get the known results [4]

$$
\begin{aligned}
q^{(K)} \equiv \frac{1}{N^{K}}\left\langle\prod_{e=1}^{K} \sum_{i_{e}=1}^{N} \sigma_{i_{e}} \mu_{i_{e}}\right\rangle=\frac{1}{N^{K}}\left(\prod_{e=1}^{K} \sum_{i_{e}=1}^{N}\right)\left\langle\prod_{e=1}^{K} \sigma_{i_{e}}\right\rangle^{2}= \\
\quad=\int_{0}^{1} \mathrm{~d} x q^{K}(x)=\int_{q_{\mathrm{m}}}^{q_{M}} \mathrm{~d} q P(q) q^{K} .
\end{aligned}
$$

It is important to note that in the case where the external magnetic field is strictly zero the function $\left.P(q)\right|_{h=0}$ is different from the function $\left.P(q)\right|_{h=0^{+}}$in an infinitesimal small magnetic field : only in the first case two configurations with all the spin reversed are allowed; we easily obtain :

$$
\left.P(q)\right|_{h=0}=\frac{1}{2}\left[\left.P(q)\right|_{h=0^{+}}+\left.P(-q)\right|_{h=0^{+}}\right]
$$

Indeed general symmetry requirements imply that at $h$ strictly zero, $P(q)$ is a symmetric function of $q[5]$.

It is known [1] that in the infinite range mode $q(0)$ is proportional to $h^{2 / 3}: q_{m}$ will thus be positive as soon $h \neq 0$ and will become equal to $-q_{\mathrm{M}}$ at $h$ strictly equal to zero.

Now if we study the time evolution of the system it is natural to suppose that the process of going from one state to another state with overlap $q$ less that $q_{\mathrm{M}}$ will take a macroscopically large time; the time being larger and larger the smaller the value of $q$ [6]. We propose that at a time $t$ (very large) the system has visited all states which have an overlap greater that $q(t)$ (only when $t$ goes to infinity $q(t)$ goes to $\left.q_{\mathrm{m}}\right):$ in other words $q(t)$ is the minimum overlap possible at time $t$.

This proposal may be expressed in more quantitative terms by defining :

$$
\begin{aligned}
& q^{(1)}(t)=\frac{1}{N} \sum_{i=1}^{N}\left[\frac{1}{t} \int_{0}^{t} \mathrm{~d} t^{\prime} \sigma_{i}\left(t^{\prime}\right)\right]^{2} \\
& q^{(2)}(t)=\frac{1}{N^{2}} \sum_{i=1}^{N} \sum_{j=1}^{N}\left[\frac{1}{t} \int_{0}^{t} \mathrm{~d} t^{\prime} \sigma_{i}\left(t^{\prime}\right) \sigma_{j}\left(t^{\prime}\right)\right]^{2} \\
& q^{(3)}(t)=\frac{1}{N^{3}} \sum_{i=1}^{N} \sum_{j=1}^{N} \sum_{e=1}^{N}\left[\frac{1}{t} \int_{0}^{t} \mathrm{~d} t^{\prime} \sigma_{i}\left(t^{\prime}\right) \sigma_{j}\left(t^{\prime}\right) \sigma_{e}\left(t^{\prime}\right)\right]^{2} \\
& q^{(K)}(t)=\frac{1}{t^{2}} \int_{0}^{t} \mathrm{~d} t_{1} \int_{0}^{t} \mathrm{~d} t_{2}\left[\frac{1}{N} \sum_{i=1}^{N} \sigma_{i}\left(t_{1}\right) \sigma_{2}\left(t_{2}\right)\right]^{K}
\end{aligned}
$$

It is clear that when $t$ goes to infinity the $q^{(K)}(t)$ go to the $q^{(K)}$ of equation $4:$ according to the previous arguments we should have in the region of large times

$$
q^{(K)}(t)=\int_{q(t)}^{q_{M}} q^{K} P(q) \mathrm{d} q / \int_{q(t)}^{q_{M}} P(q) \mathrm{d} q .
$$

According to this hypothesis all the functions $q^{(K)}(t)$ depend on only two functions $P(q)$ and $q(t)$, the first being already computed in the infinite range model [1].

At $h$ strictly equal to zero all the $q^{(K)}(t)$ with odd $K$ go to zero when $t$ goes to infinity.

If we consider finite dimensional systems it is possible that the function $P(q)$ defined in equa- 
tions 2, 3 is zero in the infinite volume limit : in this case no glassy phase should exist. The formalism discussed here may be still useful : we introduce a function $P_{L}(q)$ defined as

$$
\left\langle\exp \left(y \frac{1}{L^{0}} \sum_{i} \sigma_{i} \mu_{i}\right)\right\rangle=\int_{q_{\mathrm{m}}}^{q_{\mathrm{M}}} \mathrm{d} q P_{L}(q) \exp (y q)
$$

where the sum over $i$ runs inside a $D$-dimensional cube of side $L$. Although at sufficient low dimensions the function $P_{L}(q)$ goes to zero when $L$ goes to infinity at $q \neq 0$, it may be interesting to study the function $P_{L}(q)$ for finite but large $L$ and to try to connect the behaviour of this function with the slow approach to equilibrium of these systems (e.g. in two dimensions no phase transition is present and the time to approach the equilibrium is quite large but finite) [7]. More modestly in the infinite range model it would be very interesting to derive (7) using the formalism of reference 6 (or alternatively derive the formalism of [6] from Eq. 7).

We stress that equations 6,7 are supposed to hold at thermal equilibrium; the comparison with the results of reference 6 is not straight forward : indeed it was shown in reference 8 that the formalism of [6] is more suited to study the approach to equilibrium.

A computer simulation to verify the correctness of equations 4 and 7 should not require too much CPU time in the case of the infinite range model (the total amount of CPU time should be compared to that used in Ref. 9) and it will be clearly welcomed.

\section{References}

[1] PARISI, G., Phys. Rev. Lett. 43 (1979) 1754 ; J. Phys. A 13 (1980) L115, 1101, 1887; and Proceedings of the Les Houches Summer School, 1982, to be published.

[2] PARISI, G., The order parameter for spin glasses, submitted to Phys. Rev. Lett.

[3] De Dominicis, C., GabaY, M., Orland, H., J. Physique Lett. 42 (1981) L-522.

[4] De Dominicis, C. and Itzykson, C., unpublished.

[5] De Dominicis, C. and Young, A. P., Saclay preprint 1982 ; and SOMMERS, H. J., J. Physique Lett. 43 (1982) L-719.

[6] Sompolinsky, H., Phys. Rev. Lett. 47 (1981) 935; and SOMMERs, H. J., in On the dynamic mean field theory of spin glasses, submitted to Z. Phys. B.

[7] Young, A. P., Imperial college preprint, 1983.

[8] De Dominicis, C. and Young, A. P., J. Phys. A Lett., to be published (1983).

[9] Mackenzie, N. D. and Young, A. P., Phys. Rev. Lett. 49 (1982) 301. 